\title{
CAMPAMENTOS RESIDENCIALES TARDÍOS A CIELO ABIERTO EN EL ALTO RÍO CISNES (REGIÓN DE AISÉN): APPELEG 1 (CIS 009)
}

\author{
HÉCTOR VELÁSQUEZ;, CÉSAR MÉNDEZ**, OMAR REYES",**, VALENTINA TREJO, \\ LORENA SANHUEZA", DANIEL QUIROZ","**" Y DONALD JACKSON"*
}

\begin{abstract}
RESUMEN
Se expone el estado de las investigaciones realizadas en un contexto a cielo abierto en el segmento alto del valle del río Cisnes. Appeleg 1 corresponde a un asentamiento superficial multicomponente con una significativa diversidad de materiales que, en su mayoría, se ubica temporalmente dentro de los últimos 1.500 años. Se presenta el contexto del sitio, la metodología de recuperación de datos espaciales y los análisis llevados a cabo con los materiales recuperados. Finalmente, se discuten algunos problemas sustentados sobre la información obtenida y proyecciones de la investigación en este tipo de sitios.
\end{abstract}

PALABRAS CLAVES: Holoceno Tardío, Patagonia Central, sitios a cielo abierto, campamentos residenciales.

\section{LATE OPEN AIR RESIDENTIAL CAMPS AT UPPER RÍO CISNES (AISÉN REGIÓN): APPELEG 1 (CIS 009)}

\begin{abstract}
We present the results of ongoing research of an open-air site in the upper Cisnes river. Appeleg 1 is a multicomponent open air settlement dating to the last 1.500 years that shows a significantly diverse artefact assemblage. We present the context of the site, methods employed for recording and collecting spatial data, and the results of different analysis conducted on recovered material. We discuss some of the issues raised by the dataset and examine the potential of the methodology to investigate other open-air sites.
\end{abstract}

KEY WORDS: Late Holocene, Central Patagonia, open-air sites, residential camps.

\footnotetext{
* Centro de Estudios Humanos y Patrimoniales, Santiago.

* Departamento de Antropología, Facultad de Ciencias Sociales, Universidad de Chile.

*** Centro de Estudios del Hombre Austral, Instituto de la Patagonia, Universidad de Magallanes.

**** Centro de Documentación de Bienes Patrimoniales, DIBAM.
} 


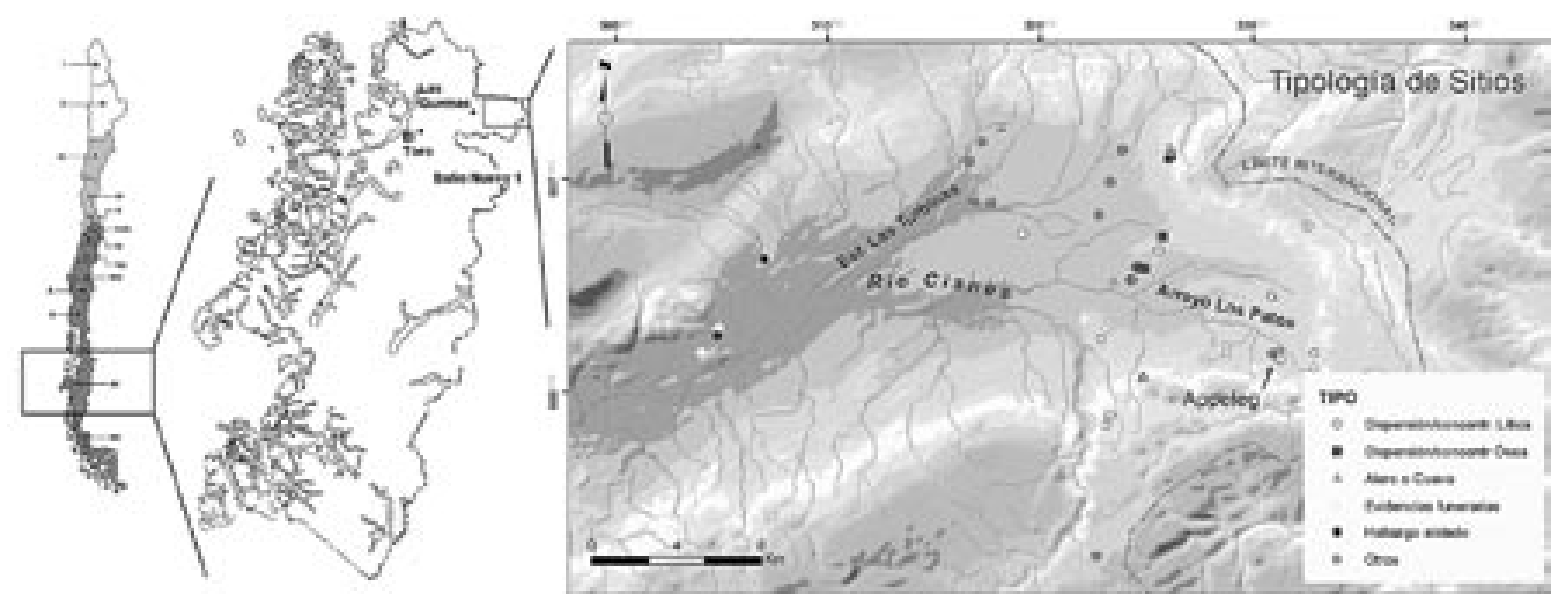

Fig. 1. Mapa del área de estudio.

\section{INTRODUCCIÓN}

Uno de los aspectos poco estudiados arqueológicamente en Patagonia central son los asentamientos residenciales a cielo abierto. Por el contrario, sitios bajo reparo u otros a cielo abierto como cementerios y talleres son frecuentemente reseñados (p.e. Castro y Moreno 2000, Goñi 2000, Goñi y Barrientos 2000, Mena 2000, Figuerero 2004, Bellelli 2005). En este sentido, parte de los objetivos centrales del equipo de investigación de la cuenca del río Cisnes $\left(\sim 44^{\circ}\right.$ S) era documentar las ocupaciones a cielo abierto del Holoceno Tardío final (e históricas) que pudie- sen encontrarse en el segmento superior del valle. Las interpretaciones relativas a los asentamientos no permanentes de grupos "tehuelche" -últimos momentos de la secuencia ocupacional regional (Mena 2000, Martinic 2005)- son fundamentales para generar modelos de uso del espacio. El trabajo presentado propone una puesta al día de los análisis conducidos en un asentamiento significativo -Appeleg 1 (Fig. 1)-cuya extensión, componentes materiales, dataciones y contexto cultural, nos permiten sugerir que correspondería a un asentamiento residencial a cielo abierto. Presentamos la metodología involucrada en la recopilación de datos, especialmente lo

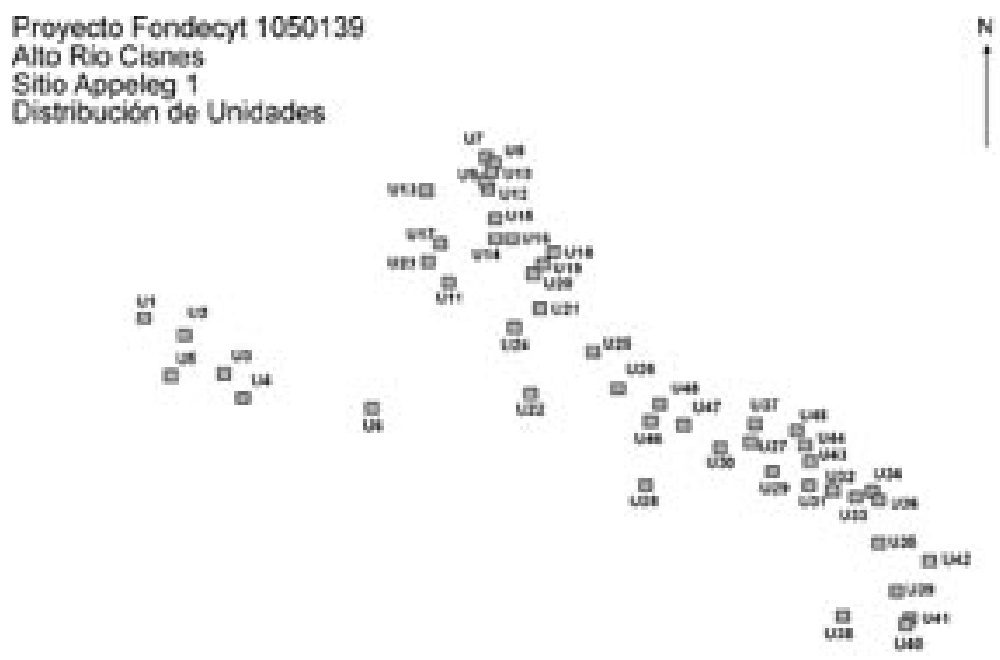

Fig. 2. Disposición de las unidades de concentración de materiales en Appeleg 1 (CIS 009). 


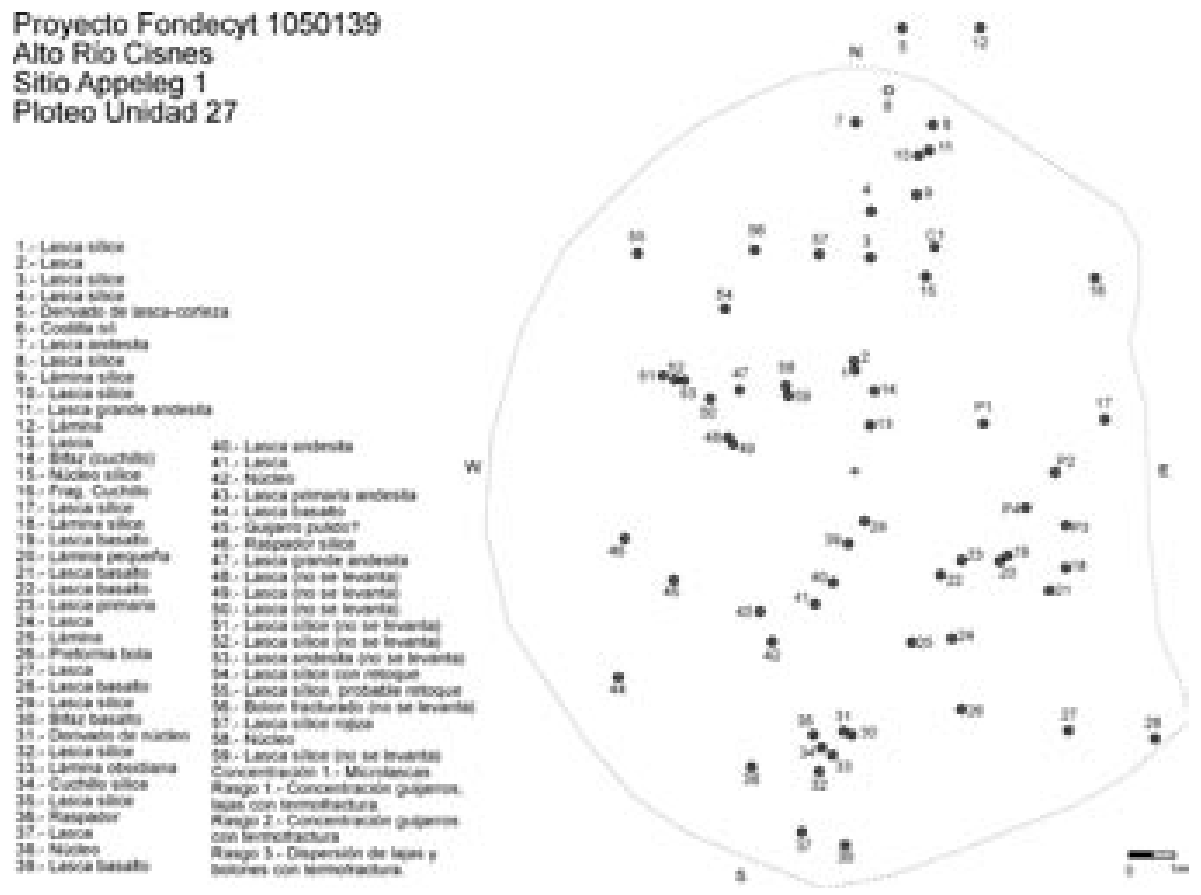

Fig. 3. Metodología de mapeo sistemático, unidad 27, Appeleg 1 (CIS 009).

concerniente a la información espacial recuperada en terreno, los análisis de los distintos materiales y las dataciones absolutas obtenidas en el sitio. El objetivo central de este trabajo es sentar las bases para futuras instancias interpretativas relativas al uso del espacio interno de los campamentos, las dinámicas socio-culturales dentro de los últimos 1.500 a 1.000 años y la relación con otros sitios contemporáneos en la región inmediata y en áreas más lejanas.

Dada la extensión e intensidad de uso de esta particular área, se procedió a trabajar por medio de la discriminación de unidades independientes, definidas por la concentración de restos materiales. Se identificó un total de 49 unidades significativas (Fig. $2)$, las que fueron dimensionadas. En atención a los posibles patrones de distribución espacial, algunas de estas unidades fueron intensamente recolectadas por medio de mapeo sistemático pieza a pieza (Fig. 3). Las unidades muestreadas corresponden a un $16,3 \%$ del total (unidades 10, 15, 16, 20, 21, 27, 37 y 49) y contemplaron la selección de sectores con material lítico, cerámico e histórico. Ello, en conjunto con los análisis de materiales, contribuyó a la definición de función de sitio. A fin de determinar la cronología de las ocupaciones, y en atención a la falta de muestras orgánicas, se llevaron a cabo dos dataciones por termoluminiscencia, una en cerámica y la segunda en una roca de fogón con alto contenido de cuarzo (Tabla 1, Fig. 4). Adicionalmente,

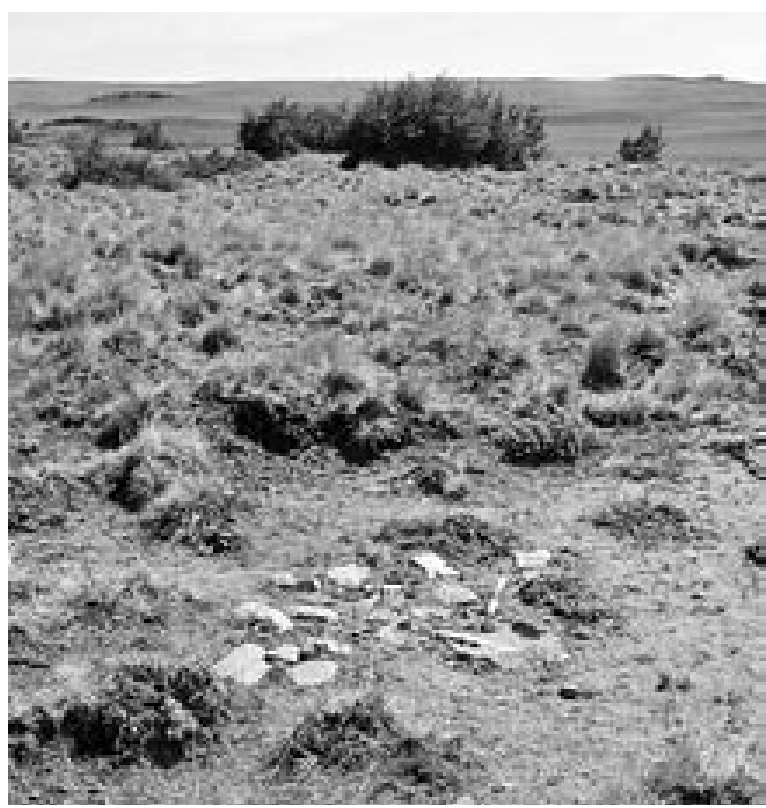

Fig. 4. Rasgo expuesto en la unidad 7 de Appeleg 1 (CIS 009), el cual fue fechado. 
Tabla 1. Dataciones de Appeleg 1, Laboratorio de Radiactividad y Termoluminiscencia de la Facultad de Física de la Universidad Católica de Chile.

\begin{tabular}{c|lcccc}
\hline MUESTRA & \multicolumn{1}{|c}{ DESCRIPCIÓN } & $\mathrm{P}(\mathrm{Gy})$ & $\mathrm{D}(\mathrm{Gy} / \mathrm{año})$ & EDAD (años AP) & FECHA \\
\hline UCTL 1805 & Roca de fogón, unid. 7 & $2,75 \pm 0,30$ & $2,04^{*} 10^{-3}$ & $1290 \pm 130$ & $660 \mathrm{DC}$ \\
UCTL 1806 & Cerámica, unid. 5 & $2,37 \pm 0,20$ & $2,99 * 10^{-3}$ & $740 \pm 80$ & 1210 DC \\
\hline
\end{tabular}

los análisis pretendieron vías posibles de asignación de cronología relativa.

\section{EL ASENTAMIENTO APPELEG 1 (CIS 009)}

El sitio Appeleg 1 (Figs. 1 y 5) corresponde a un agregado de concentraciones de restos arqueológicos de gran distribución espacial $\left(14.500 \mathrm{~m}^{2}\right.$, Reyes et al. 2006). Se caracteriza por la presencia de unidades discretas de material lítico, óseo, cerámica, vidrio y metal (en distintos tipos de asociaciones), las que fueron designadas como unidades independientes a fin de mantener un control espacial riguroso. En sectores puntuales fue posible identificar también agrupaciones intencionales de piedras y vestigios de áreas de combustión con rocas a modo de emplantillados (Fig. 4). Las evidencias se encuentran principalmente en superficie, aunque fue posible observar algunos sectores con potencial estratigráfico menor.

El sitio se ubica en un extenso sector de dunas en deflación sobre una planicie abierta (Fig. 5) protegida en el Norte por un bosque de ñire (Nothofagus antartica). El ambiente es de estepa con un clima frío seco, en donde las precipitaciones no superan los $400 \mathrm{~mm}$ anuales. La vegetación dominante es esteparia con componente arbustivo,

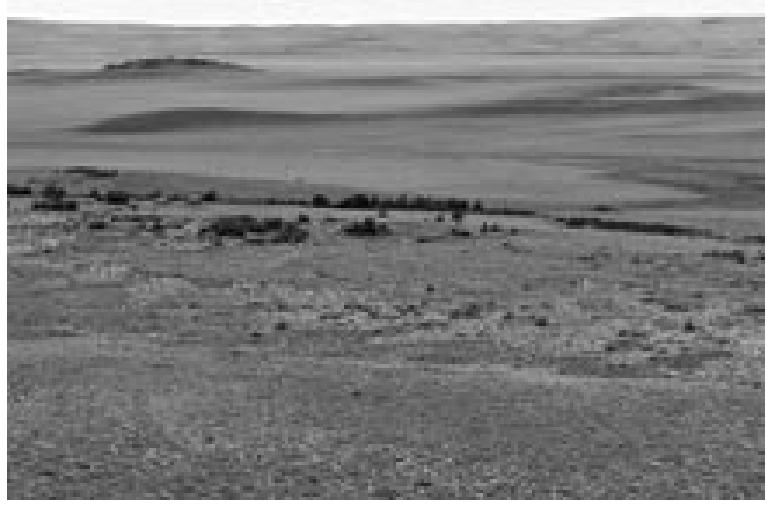

Fig. 5. Entorno de Appeleg 1 (CIS 009). compuesta principalmente por Vicia speciosa, Stipa sp., Festuca sp. y Erodium cicaterium, y en menor medida por Nothofagus antarctica y N. pumilio, en asociación a Acaena sp., Ribes sp., Berberis sp. y Chiliotrichium diffusum. El sitio se asocia a un ojo de agua, que corresponde a la fuente más próxima de aprovisionamiento, a $100 \mathrm{~m}$ de distancia (Reyes et al. 2006).

El evidente efecto palimpsesto observado en este sitio sería el resultado de una serie de agentes medioambientales y antrópicos que habría incidido en la formación, distribución y posterior coadunación de las evidencias materiales (Schiffer 1996) generando un registro promediado. Tal como se señalara, Appeleg 1 se encuentra emplazado sobre un sistema de dunas deflacionadas, cuyas características de matriz arenosa, sumadas a la acción del viento, provocaron la movilización de los materiales vertical y horizontalmente (para lo cual se implementó el registro espacial pieza a pieza). Dentro de los factores antrópicos de mayor relevancia, se encuentra la actividad ganadera ovina intensiva desde principios del siglo XX (1906: establecimiento de Estancia Río Cisnes), la cual ha generado rutas de tráfico aledañas al sitio (caminos chateros), pisoteo de animales, aprovechamiento del bosque de ñire y levantamiento selectivo de materiales arqueológicos. Estos procesos se hacen evidentes a través de la presencia del sitio Appeleg 3 y la ruta chatera que se le asocia, ambos de clara adscripción colono-estanciera y emplazados a pocos metros de Appeleg 1.

\section{LOS CONJUNTOS LÍTICOS}

El material lítico corresponde al conjunto de evidencias cuantitativamente dominante en el contexto de Appeleg 1. Se encuentra representado en variadas categorías morfo-funcionales, tecnológicas y materias primas, en casi la totalidad de las unidades observadas. Las piezas recuperadas, pese a estar en superficie, exhibieron niveles muy homogéneos de patinación, que a su vez son diferentes de sitios 
próximos como El Deshielo (CIS 008), contexto con excepcional pátina (probablemente más temprano) en los conjuntos del área de estudio (Méndez et al. 2007). Las inferencias logradas por el análisis apuntan hacia un conjunto más bien homogéneo, que se condice con lo esperable para un registro material de campamentos residenciales con una multiplicidad de actividades domésticas representadas. En términos tipológicos, si bien se observaron indicadores que regionalmente se asocian a una temporalidad amplia, la gran mayoría de las piezas puede ser considerada como parte de una unidad cultural tardía en el área (Méndez et al. 2007).

Las materias primas dominantes son múltiples variedades de sílices, y en menor medida algunas rocas basálticas de calidad media. Las sílices probablemente correspondan a rocas obtenidas en una amplia área de aprovisionamiento aún indeterminada. Ello se sugiere dada su ausencia en el entorno inmediato (Méndez et al. 2007), la variabilidad que presentan y el hecho que se encuentren representadas por instrumentos formatizados (principalmente raspadores y en menor medida implementos de corte y puntas de proyectil), y desechos de talla de las etapas finales de la secuencia de reducción. Las rocas basálticas se obtuvieron localmente en forma de rodados, como lo atestiguan algunas evidencias con remanentes de corteza. A lo anterior se suma más de un tipo de obsidiana, entre lo que presumiblemente habría la variedad de Pampa del Asador (Molinari y Espinosa 1999, Espinosa y Goñi 1999, Mendez et al. 2007), aunque en todos los casos en muy baja representación. Se observó muy poca evidencia de materias primas de granulometría gruesa, aunque se destaca la presencia de algunos guijarros porosos volcánicos transportados al sitio y algunas rocas graníticas empleadas como manos de moler.

Las evidencias recuperadas corresponden principalmente a segmentos de cadenas operativas incompletas, ya que la mayoría son derivados de núcleo; aunque ello bien podría estar sesgado por los factores tamaño y visibilidad. Se observó -en menor cuantía- desechos de desbaste bifacial, con la excepción de la unidad 37, donde son frecuentes. Aún así las evidencias obtenidas en el sitio no son suficientes como para pensar en áreas de manufactura bifacial, sino prioritariamente el ingreso de piezas avanzadas o terminadas. Ya que son frecuentes los instrumentos formales en el sitio, su descarte debió vincularse a necesidades de reemplazo una vez agotadas sus vidas útiles. Sólo en un caso fue posible identificar una cadena operativa más completa -con algunos reensamblajes (unidad 21) - en la forma de grandes extracciones de andesita, para la manufactura de instrumentos expeditivos sin modificaciones.

En el sitio se observó la integración de diversas soluciones tecnológicas con una significativa recurrencia en las unidades muestreadas. Son destacables las evidencias de tecnología laminar, sobre todo en la forma de extracciones de series finales e instrumentos propiamente tales, ya que los núcleos son infrecuentes. Su tamaño general es siempre pequeño y su manifestación principal se encuentra en la forma de raspadores frontales y terminales extraídos desde núcleos unidireccionales y hojas con y sin modificación marginal. Ambos grupos están manufacturados en sílices de alta calidad. La talla bifacial, se representa por puntas de proyectil, bifaces/preformas y cuchillos bifaciales finamente manufacturados. Ambas soluciones, tecnología laminar y bifacial, corresponden a los principales exponentes de los instrumentos formales -diseño estandarizados y vida útil extendida- observados en el asentamiento. No obstante, los conjuntos formales se encuentran frecuentemente asociados a conjuntos informales de rápido descarte. Ello constituye una excepción, ya que en el resto de los sitios observados en el sector alto del valle, esta integración tecnológica (que es por lo demás muy común) se encuentra raramente representada. La presencia de una mayor variabilidad de soluciones tecnológicas podría estar implicando una mayor permanencia en el asentamiento, a diferencia de otros campamentos más pequeños y transitorios.

A las categorías morfo-funcionales ya reseñadas (puntas de proyectil, raspadores frontales, Fig. 6 a-g, láminas usadas) se suman múltiples tipos de herramientas en proporción minoritaria, aunque igualmente destacables. Raederas y cuchillos bifaciales se observaron en bajas frecuencias, lo que sugiere que probablemente fueron reemplazados por medio del uso de los filos vivos de hojas u otras extracciones, cuyas capacidades de desempeño son probablemente superiores. Sobresale el hecho que -al menos-algunas raederas y cuchillos bifaciales también hayan sido manufacturados a partir de láminas, especialmente las de series iniciales (más espesas). Pese a su baja representación, las manos de moler constituyen una 


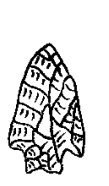

a

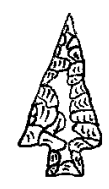

b

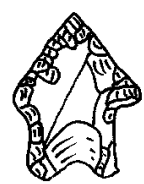

c

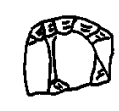

d

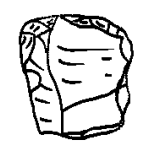

e

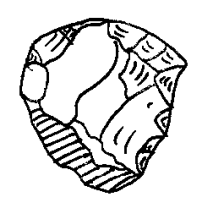

$f$
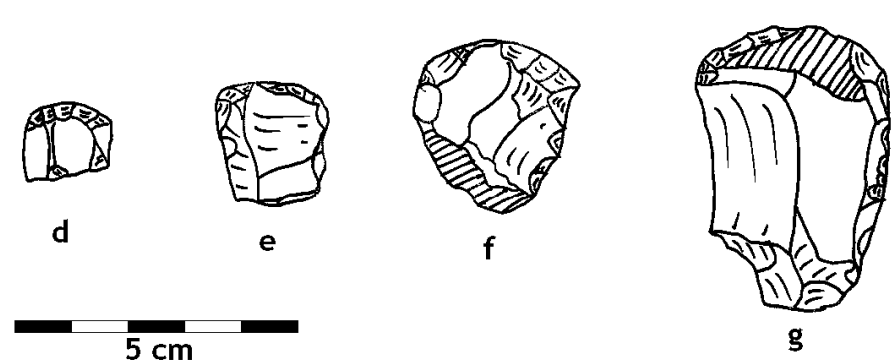

g
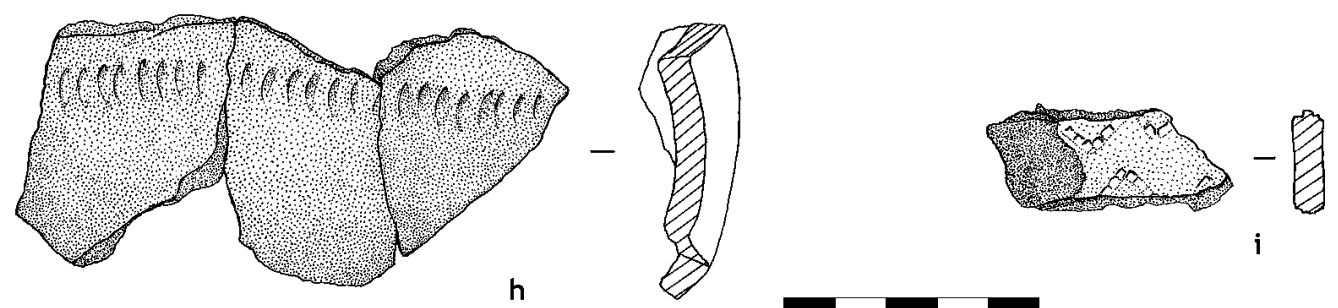

i

Fig. 6. Materiales diagnósticos. Referencias: a. - g.: material lítico, h. - i.: cerámica. Descripción: a. - c. puntas de proyectil de la unidad tardía, d. - g. variabilidad de raspadores frontales, h. - i. cerámica monocroma decorada con incisiones.

categoría destacable, especialmente si consideramos su pertinencia a contextos de actividades domésticas y su baja frecuencia en Patagonia. En Appeleg 1 se manifiestan poco formatizadas, lo que sugiere un uso minoritario. En cuanto a las categorías líticas subrepresentadas o ausentes sobresale la ausencia de muescas y denticulados, así como, la muy baja a nula frecuencia de instrumentos sobre núcleo para la apropiación de maderas (como cepillos, tajadores y machacadores) y la bajísima presencia de percutores y sobadores.

La información obtenida nos permite sugerir una función preferentemente residencial para las unidades muestreadas y para el asentamiento en general. La prioridad de categorías morfo-funcionales atribuidas a la realización de actividades domésticas, que involucran principalmente el procesamiento $y$ consumo de recursos, aboga en favor de esta idea. Adicionalmente, el hecho que gran parte de los instrumentos ingrese en estado plenamente funcional al asentamiento, permite inferir uso, por sobre manufactura. En términos generales, esta observación es extensible a la gran mayoría de los conjuntos líticos de las unidades muestreadas. De forma minoritaria se identificó indicadores de tecnología de apropiación, bolas líticas y puntas de proyectil, las cuales se inter- pretan como depositadas en el ámbito habitacional en atención a posibles conductas de reemplazo en las armas arrojadizas. Un ejemplo claro de la función residencial constituye la unidad 37, donde de las 64 piezas mapeadas con referencia espacial única, 27 corresponden propiamente a instrumentos retocados (42\%). Resulta una cifra bastante significativa que indica un contexto en donde se llevaron a cabo múltiples actividades. Excepción constituye la unidad 27 (Fig. 3) en donde grandes lascas de basalto y andesita, de aprovisionamiento inmediato, y otras materias primas fueron procesadas en la manufactura de instrumental. De confirmarse una eventual sincronía, esta área bien podría tener una función de taller asociada a las demás unidades para el sitio; generando por tanto, distinciones en el uso del espacio al interior del área de campamento.

Aun con la baja capacidad diagnóstica que permite el análisis lítico, sobre la base de los indicadores presentes es posible sugerir una asignación cronológica tardía para la mayoría de los conjuntos reconocidos en el sitio. La asociación entre pequeños a medianos raspadores frontales, la tecnología de láminas de tamaño pequeño y la presencia de varias morfologías de puntas de proyectil triangulares con aletas y pedúnculo (Fig. 6 a-c), en atención a los 
antecedentes regionales (Mena y Jackson 1991, Mena 2000, Méndez y Velásquez 2005, Méndez et al. 2007), nos permiten suponer que el asentamiento se ubique temporalmente dentro de los últimos 1.500 a 1.000 años. Las dataciones absolutas obtenidas (Tabla 1) corroboran dicha apreciación. La presencia, tanto de puntas de proyectil triangulares delgadas de pedúnculo pequeño y aletas, como triangulares de pedúnculo ancho y alteas laterales (ligeramente más grandes, Fig. 6), si bien ha sido explicada como una eventual diacronía, no necesariamente responde a un palimpsesto de dos momentos independientes, sino alternativamente a la manutención de distintas tecnologías (Ratto 1994). Cabe notar, no obstante, la presencia de material lítico característico de cronologías más tempranas en el asentamiento. Un lito geométrico y un raspador de gran tamaño (con una intensa patinación que lo diferencia del resto de las evidencias líticas), similar a los del nivel temprano de Cueva Fell (Jackson y Méndez 2007, Méndez et al. 2007) y algunas puntas de proyectil triangulares de base convexa, sugieren que es posible la presencia de otros componentes más tempranos, aunque minoritarios, en el sitio.

\section{EL CONJUNTO ARQUEOFAUNÍSTICO}

Los restos óseos registrados son muy escasos y se presentan en muy mal estado de preservación debido al carácter superficial del sitio. Adicionalmente, es necesario considerar que los restos no necesariamente correspondan a vestigios de subsistencia de las ocupaciones humanas, sino que factores de incorporación natural estén interviniendo la muestra. Se reconoció la presencia de varias unidades anatómicas pertenecientes a especies introducidas, como caballo y vacuno y a taxones nativos, como guanaco y ñandú. No se pudo determinar agentes de faenamiento debido a la alta meteorización de las superficies de las evidencias, no obstante, en un caso, la presencia de una sección facetada sugiere acción de un instrumento filoso. En este sentido, de ser parte del conjunto aprovechado, el registro vacuno y equino asociado a guanaco y ñandú, darían cuenta de la coexistencia de una economía ganadera y un sustrato cazador-recolector. De hecho la incorporación del caballo en Patagonia, redujo considerablemente los costos asociados a transporte y condujo a importantes readaptaciones en los sis- temas de asentamientos y subsistencia, facilitando, también, la adopción de nuevos elementos culturales (Palermo 1986, 1988, Mandrini 1992).

\section{CERÁMICA}

En Appeleg 1 se recuperó un total de 44 fragmentos cerámicos, provenientes de dos unidades de recolección, concentrándose prácticamente todo el material en la № 5, ya que en la unidad 49 sólo se recuperó un fragmento (Fig. 6 h-i). Dado que la presencia de tal cantidad de material cerámico en un sitio de la Región de Aisén es excepcional, se consideró relevante realizar un análisis lo más exhaustivo posible, pretendiendo una caracterización de las superficies, espesores, pastas y decoraciones, la inferencia de las categorías formales presentes y el número de vasijas representadas. De la misma manera se consignó la presencia de hollín y ciertas características de la superficie de los fragmentos, producida por procesos postdepositacionales.

El material recuperado se presenta muy homogéneo en términos del tratamiento de las superficies, ya que todos los casos son alisados, tanto por el exterior, como por el interior. En cuanto a los colores, éstos varían principalmente en la gama de los café (claro a oscuro), aunque algunos pocos fragmentos presentan sus superficies exteriores de color anaranjado. La variabilidad observada al interior de cada fragmento sugiere que los tonos cafés pueden ser resultado de la exposición de las vasijas al humo y por lo tanto, al fuego. El espesor de los fragmentos es bastante homogéneo y varía en un rango de 4.3 a $6.5 \mathrm{~mm}$. Esto queda reflejado en el promedio $(5.28 \mathrm{~mm}$ ) y su escasa desviación estándar $(0.47 \mathrm{~mm})$. Algunos fragmentos presentan hollín adherido, que siempre está localizado en las superficies interiores. Una característica postdepositacional relevante es la pérdida de materia, tanto arcilla como antiplástico, o "delaminado", que se reconoce en 11 fragmentos.

La mayor parte de los fragmentos recuperados corresponde a cuerpos, aunque pudieron identificarse además 9 cuellos, un borde, un labio y un borde dudoso. Las características de estos fragmentos nos sugieren la presencia de vasijas restringidas con cuello hiperboloide y borde con labio plano, o bien borde evertido. El diámetro medido permite inferir vasijas de tamaño mediano (150 mm). 
El análisis de pastas se vio dificultado porque al realizar cortes frescos se descubre una matriz negra que impide observar los áridos. Esto puede deberse a arcillas con alto contenido de materia orgánica o bien cocción en ambiente reductor. No obstante, se pudieron observar dos tipos característicos. Un patrón de áridos blancos y traslúcidos opacos "teñidos" de amarillo/anaranjado, de forma subredondeada y subangular, tamaño heterogéneo (dentro de un rango mediano/pequeño) y densidad alta, con matriz color negro. Un segundo patrón corresponde a matriz color café claro con inclusiones de rocas color café claro/beige de tamaño grande y en densidad media/baja. El primer patrón incluye a todos los fragmentos revisados, excepto el fragmento decorado de la unidad 49, cuya pasta pertenece al segundo patrón.

De acuerdo a las decoraciones, colores de las superficies, formas y pastas, los fragmentos del sitio Appeleg 1 corresponderían a un mínimo de 3 vasijas representadas por aquélla con decoración impresa escalerada de la unidad 49, una vasija con cuello y labio plano y una pieza con borde evertido, pudiendo los fragmentos con decoración impresa en el cuerpo de la unidad 5 y otros fragmentos con cuerpo anaranjado, eventualmente corresponder a otras dos vasijas.

Los decorados corresponden a lo que técnicamente se conoce como "impresiones", considerada como técnica decorativa plástica, definida por la impronta de un objeto sobre la arcilla fresca. Los fragmentos recuperados de la unidad 5 presentan una hilera de impresiones semicirculares paralelas, que muy probablemente corresponde a la impronta de una uña (inciso unguiculado de surco rítmico, Fig. 6h). El fragmento recuperado de la unidad 49 presenta un motivo escalerado, formado por un continuo de impresiones poco profundas realizadas con un objeto que tenía su extremo distal plano, de sección cuadrangular (Fig. 6i).

Tradicionalmente se asocia la cerámica de Patagonia a una influencia cultural proveniente del área mapuche occidental, como parte de la reconfiguración poblacional tardía (Casamiquela 1969). No obstante, los fragmentos con decoración incisa "rítmica" encontrados en Appeleg 1, se asemejarían más a la cerámica de la zona pampeana argentina (Aldazabal 1993, 1999, Politis et al. 2001, González 1997, Berón 2003). En la Araucanía, a pesar que la decoración incisa es muy variada, tanto tipológica, temporal (Pitrén a El Vergel), como espacialmente, el tipo "inciso rítmico" nunca ha sido registrado hasta la fecha (Dillehay 1989).

Una revisión del registro de cerámica en Patagonia, indica que su presencia es muy escasa (Orquera 1987, Mena y Jackson 1991 y Borrero 1994-5). La fecha más antigua para la introducción de cerámica en la Patagonia, proviene del sitio Los Cipreses, situado en la provincia de Neuquén (Silveira 1996), con fecha de 1.500 AP, asociada al componente cerámico I, que posee fragmentos claramente Pitrén (pintura negativa negro y rojo). Con una fecha ligeramente más temprana ( 1.960 $\mathrm{AP})$, se registra en la costa de la bahía de San Blas (Patagonia nororiental), cerámica decorada muy escasa, que evidencia la utilización de técnicas de inciso de línea, surco rítmico y punteado (Eugenio y Aldazabal 2004). Este tipo de cerámica se encuentra presente, aunque no muy abundantemente, en todo el litoral marítimo pampeano durante el Holoceno Tardío (Bonomo 2005). Según algunas hipótesis, su incorporación por parte de las sociedades patagónicas se debería a una creciente escasez relativa de alimentos debido a un aumento de la población, pues permite un aprovechamiento más intensivo de los recursos (Mena y Jackson 1991), siendo su uso irregular, no adaptativo, sin alcanzar un éxito replicativo, por lo que su frecuencia es relativamente baja (Borrero 1994-5, Senatore 1996).

En relación a la procedencia de la cerámica en Patagonia, Mena (1998) sugiere influencias no sólo de origen andino, sino también de la tradición Tupí-Guaraní de los alrededores del río de la Plata, que pudieron haber llegado por rutas que rodearan la Patagonia noroccidental. Por su parte, Bellelli (1980) señala que la dispersión de la cerámica en la Patagonia habría ocurrido por la costa atlántica, de norte a sur. Por otro lado, Orquera (1987:398) indica que "los raros motivos decorativos (incisos y punzonados) manifiestan cierto parecido con los de Pampa y la cuenca del Plata".

En la región de Pampa y Patagonia, Politis y colaboradores (2001) han establecido tres momentos para la incorporación de la alfarería: (a) entre 2.500 y 3.000 años AP en el sudeste de la Región Pampeana y el norte y noreste de Uruguay; (b) entre 1.700 y 1.500 años AP en el noreste de la Región Pampeana y en el litoral del Río de la Plata; 
y (c) entre 1.200 y 1.500 años AP en Patagonia Septentrional. Nuestros resultados han situado la cerámica en la Región de Aisén al menos en 740 AP (Tabla 1), más tempranos que los resultados obtenidos previamente en 4S-3 (405 AP) en el valle de río Ibáñez (Mena y Lucero 2004).

\section{LAS EVIDENCIAS HISTÓRICAS}

Las evidencias históricas materiales están constituidas únicamente por restos de vidrio y metal, las que en algunos casos se han registrado asociadas superficialmente a materiales líticos, alfarería y restos óseos. El conjunto de restos de vidrio incluye un total de 105 piezas; 4 completas o casi completas y las restantes 101 fragmentos de golletes, cuerpos y bases. Si bien se realizaron algunos ensamblajes de fragmentos, la alta frecuencia, tamaño y características de cuerpos no se corresponden con la baja frecuencia de golletes y bases, lo que sugiere numerosas piezas faltantes, las que seguramente aún no han sido expuestas por los procesos de deflación que afectan el sitio.

Los colores de los fragmentos de vidrio (lo que se relaciona a su composición química), incluyen vidrio verde claro, verde oscuro, café y variedades transparentes, este último a veces con algunas tonalidades de rosado. Por otra parte, las características de forma, tamaño y color de los restos indican la presencia únicamente de botellas de alcohol y frascos. Estos últimos corresponden al menos a 6 piezas identificadas por tres tipos de bases, un gollete $y$ dos fragmentos de cuerpo, uno de ellos con una inscripción incompleta bajo un círculo. Tres piezas corresponden a vidrio transparente y las otras a vidrio de color verde claro. En todos estos casos se trata de frascos cilíndricos pequeños de vidrio grueso, probablemente correspondiente a envases de medicamentos y/o productos similares.

Por otro lado, la presencia de metal en Appeleg 1 , es efímera pero significativa y se compone básicamente por muestras de varios tipos de latas de conserva, dos cartuchos de balas y una cuchara. El estudio de las latas de conserva permitió identificar distintos tipos de envases según forma, tamaño, tipo de soldaduras e inscripciones. El conjunto de estas evidencias históricas en el sitio de Appeleg 1, indica con claridad una reocupación esporádica del asentamiento, por parte de colonos.
Algunos indicadores cronológicos (Kendrick 1967, Hunt 1975), nos permiten señalar que la ocupación histórica debió ocurrir aproximadamente entre 1880 y 1920 . No se registraron botellas con huellas de pontil, cicatriz circular de vidrio crudo y afilado, situado en las bases de botellas. Tampoco se observaron golletes hechos a mano, ni huellas de molde, lo que excluye ocupaciones previas a 1880. La presencia de huellas de molde en el cuerpo y golletes de botellas, y la factura a mano de labios, indican al menos la presencia de algunas botellas fabricadas hacia 1880. Por otra parte, la presencia de latas de conservas soldadas indica una ocupación previa a 1920, fecha en la cual se comienza con la soldadura de latas de conserva por pliegue o remache (Rojas 1996).

Por otro lado, las diferencias de pátinas, desgaste y estriamiento sobre los vidrios de botellas y frascos sugieren al menos dos eventos ocupacionales ocurridos entre 1880 a 1920 . Sólo una botella con huellas de molde y de color transparente indicaría factura industrial con posterioridad a 1930, probablemente vinculada a la producción de la Fábrica Nacional de Vidrio fundada en 1902 (Rojas y Rojas 2000). Finalmente, algunos frascos y botellas con inscripciones en inglés son productos fabricados en el extranjero y corresponden por tanto, a artículos importados y comercializados en Chile.

No se registraron otros indicadores culturales, como raspadores de vidrios o restos de metales trabajados, como fue frecuente en asentamientos indígenas de Patagonia meridional (Martinic y Prieto 1986, 1988, Martinic et al. 1995, Jackson 1991a, 1991b, 1999).

\section{DISCUSIÓN Y COMENTARIOS FINALES}

El objetivo central del presente trabajo es documentar las evidencias concernientes a la tecnología, subsistencia y la data histórica presentes en el sitio Appeleg 1, con el fin de sustentar futuras instancias interpretativas; especialmente relacionadas con el uso del espacio de los campamentos y de analogía etnográfica. Lo anterior cumple el propósito de entender las evidencias arqueológicas a la luz de las observaciones etnohistóricas, o las implicancias conductuales, su relación con aspectos funcionales y sus consecuencias en el registro arqueológico de tiempos históricos en Patagonia central (Velásquez et al. 2007). 
Los sitios adscritos a tiempos tardíos en Patagonia central son variados y abundantes (Goñi 2000). Aún así, el registro de asentamientos residenciales de grandes dimensiones, como es el caso de Appeleg 1 , no ha sido referenciado hasta el momento, a diferencia de Patagonia meridional (Martinic y Prieto 1986). Consideramos, no obstante, que no necesariamente toda la evidencia representa una ocupación sincrónica (registro promediado). Más bien, un posible uso reiterado a lo largo de un amplio lapso temporal podría explicar el registro observado y la presencia de materiales de diversa índole (temporal y funcional). Es muy probable que como campamento no haya tenido la misma extensión, ni intensidad en todos los momentos de ocupación; y proponemos que sólo puede considerarse como un gran asentamiento para el momento más tardío en que fue habitado ( 1.290 a $740 \mathrm{AP}$, en adelante). Los buenos resultados con dataciones de termoluminiscencia, especialmente sobre contextos discretos como los emplantillados de rocas, en conjunto con la metodología de recuperación de información espacial, permitirán tener un mejor control a fin de aislar las evidencias de distintas temporalidades y comprender los procesos de formación de sitios.

Por otra parte, sugerimos que el sitio fue ocupado en forma estacional, pero recurrentemente. Las condiciones ambientales de estepa en altura y la marcada estacionalidad serían consistentes con conductas de repliegue hacia áreas más benignas, como los valles andinos o la estepa oriental. Aceptando dicha diferenciación en el uso del espacio, algunos sitios registrados valle abajo, donde hay presencia de recursos alternativos, podrían estar funcionalmente articulados a Appeleg 1.

De acuerdo a los análisis de materiales líticos y a la presencia de cerámica, el sitio se definió funcionalmente como un campamento residencial, aunque subrayamos que dicha manifestación no reviste una necesaria sincronía. Dadas las categorías morfo-funcionales identificadas, como alta frecuencia de raspadores, manos de moler, implementos de corte y procesamiento, en asociación a varias vasijas, es posible inferir numerosas actividades de índole doméstica. Rasgos de quema, como emplantillados de piedras, apoyarían esta idea. Asimismo, es esperable en un contexto residencial, la proximidad a recursos claves como agua y maderas, elementos presentes en Appeleg 1.
La presencia de cerámica con una técnica decorativa "inciso rítmica" en el valle del río Cisnes, es de especial importancia para entender la probable amplitud de sistemas de movilidad, contacto y alianzas en las sociedades cazadoras recolectoras. Este sistema de complejas redes de intercambio, tanto de objetos materiales como de significados culturales, nos permite vislumbrar la magnitud a escala regional durante el Holoceno Tardío (Politis y Madrid 2001, Bonomo 2005, Berón 2003). Además, el registro de materias primas líticas no locales (entre lo que se identificó varios tipos de obsidiana), apoyaría extensos rangos de movilidad. Este fenómeno es consistente con lo que se ha denominado "extensificación" o ampliación de los rangos espaciales desde asentamientos de mayor permanencia, proceso que habría acontecido desde aproximadamente los últimos mil años (Goñi 2000).

Finalmente, consideramos que el sitio Appeleg 1 , al situarse en tiempos tardíos, se enmarca en un contexto socio-cultural extremadamente complejo y escapa a cualquier caracterización étnica simple. Además en estas áreas o zonas "marginales" los procesos son de rápida transformación, conflictos, cruce de poblaciones y escasa información documental, lo que hace este fenómeno aún difícil de explicar (Aguado 2004, Velásquez et al. 2005).

\section{AGRADECIMIENTOS}

Investigación financiada por el proyecto FONDECYT 1050139. Extendemos reconocimientos a Estancia Río Cisnes y a Carabineros de Chile del puesto fronterizo Frías-Appeleg por su invaluable colaboración logística. Agradecemos al equipo de investigación arqueológica y ambiental que apoyó la Iniciativa Cisnes: Pedro Cárdenas, Manuel San Román, Pablo González, Ismael Martínez, Carolina Belmar, Claudia Quemada, Leonardo Zúñiga y Andrés Hernández.

\section{BIBLIOGRAFÍA CITADA}

ALDAZABAL, V. 1993. Algunas consideraciones acerca de la fase cerámica Punta Indio. Palimpsesto 3:7-14.

ALDAZABAL, V. 1999. El diseño de la decoración cerámica. Una vía de interpretación. En: Actas del XII Congreso Nacional de Arqueología Argentina II: 7-15. La Plata. 
AGUADO, A. 2004. La colonización del oeste de la Patagonia Central. Departamento Río Senguer, Chubut 18901919. Fondo Editorial Provincial Gobierno del Chubut, Trelew. Argentina.

BELLELLI, C. 1980. La decoración de la cerámica gris incisa de Patagonia (República Argentina). Revista do Museu Paulista XXVII:199-225.

BELLELLI, C. 2005. Tecnología y materias primas a la sombra de Don Segundo. Una cantera-taller en el valle de Piedra Parada. Intersecciones en Antropología 6:75-92.

BERÓN, M. 2003. Dinámica poblacional y estrategias de subsistencia de poblaciones prehispánicas de la cuenca Atuel-salado Chadileuvu-Curacó, Provincia de la Pampa. Tesis de Doctorado en Arqueología, Universidad de Buenos Aires, Buenos Aires. MS.

BONOMO, M. 2005. Costeando las llanuras. Arqueología del litoral marítimo pampeano. Sociedad Argentina de Antropología, Buenos Aires.

BORRERO, L. 1994-5. Arqueología de la Patagonia Central y Meridional. Palimpsesto 4:9-96.

CASAMIQUELA, R. 1969. Un nuevo panorama etnológico del área pan-pampeana y patagónica adyacente. Ediciones del Museo Nacional de Historia Natural, Santiago de Chile.

CASTRO, A. y J. MORENO. 2000. Noticias sobre enterratorios humanos en la costa norte de Santa Cruz-PatagoniaArgentina. Anales del Instituto de la Patagonia, Serie Ciencias Sociales 28:225-231.

DILLEHAY, T. 1989. Las culturas alfareras formativas del extremo sur de Chile. Gaceta Arqueológica Andina 17:101-114.

EUGENIO, E. y V. ALDAZABAL. 2004. Los cazadores recolectores del litoral marítimo del área de bahía de San Blas, Provincia de Buenos Aires. En: Contra viento y marea... Actas de las $V$ Jornadas de Arqueología de Patagonia, editado por T. Civalero, P. Fernández y G. Guraieb, pp. 687-700. Instituto Nacional de Antropología y Pensamiento Latinoamericano y Sociedad Argentina de Antropología, Buenos Aires.

ESPINOSA, S. y R. GOÑI. 1999. Viven: una fuente de obsidiana en la provincia de Santa Cruz. En: Soplando en el viento... Actas de las Terceras Jornadas de Arqueología de la Patagonia, pp. 177-188. Universidad Nacional del Comahue e Instituto Nacional de Antropología y Pensamiento Latinoamericano, Neuquén y Buenos Aires.

FIGUERERO, M. 2004. La estructuración del espacio a través del tiempo en Cerro de los indios 1 (lago Posadas, Santa Cruz). En: Contra viento y marea... Actas de las $V$ Jornadas de Arqueología de la Patagonia, pp. 557-563. Instituto Nacional de Antropología y Pensamiento Latinoamericano y Sociedad Argentina de Antropología, Buenos Aires.

GONZÁLEZ, M. 1997. Potsherd, coypo teeth, and fish bones: hunter-gatherer-fishers in the Río Salado (Pampa Region, Argentina). En: Quaternary of South America and Antarctic Peninsula 10, editado por J. Rabassa, M. Salemme y G. Politis, 255-278. Balkema Publishers, Rotterdam.

GOÑI, R. 2000. Arqueología de monumentos históricos fuera de los centros de conquista y colonización: un análisis de caso en el sur de la Patagonia. En: Desde el país de los gigantes. Perspectivas arqueológicas en Patagonia. Actas de las IV Jornadas de Arqueología de la Patagonia, tomo 1:283-296. Universidad Nacional de la Patagonia Austral, Río Gallegos.

GOÑI, R. y G. BARRIENTOS. 2000. Estudio de chenques en lago Salitroso, provincia de Santa Cruz. En: Desde el país de los gigantes. Perspectivas arqueológicas en Patagonia. Actas de las IV Jornadas de Arqueología de la Patagonia, tomo 1:161-165. Universidad Nacional de la Patagonia Austral, Río Gallegos.

HUNT, C. 1975. Death Valley: geology, ecology and archaeology. Universidad de California Press, Berkeley.

JACKSON, D. 1991a. Raspadores de vidrio en Dinamarquero: reflejo de una encrucijada cultural. Anales del Instituto de la Patagonia, Serie Ciencias Sociales 20:58-67.

JACKSON, D. 1991b. Los instrumentos de vidrio de Cuarto Chorrillo, costa de bahía Santiago, Estrecho de Magallanes. Anales del Instituto de la Patagonia, Serie Ciencias Sociales 20:69-74.

JACKSON, D. 1999. Raspadores de vidrio en un asentamiento Aónikenk en el valle del Zurdo, zona central de Magallanes. Anales del Instituto de la Patagonia, Serie Ciencias Sociales 27:175-181.

JACKSON, D. y C. MÉNDEZ. 2007. Litos discoidales tempranos en contextos de Patagonia. Magallania 35(1):75-84.

KENDRICK, G. 1967. Bottle fragmentry betray age of historical sites. El Palacio 74(2):19-24.

MANDRINI, R. 1992. Indios y fronteras en el área pampeana (siglos XVI-XIX). Balances y perspectivas. Anuario IEHS VII:59-72.

MARTINIC, M. 1995. Aónikenk. Historia y cultura. Universidad de Magallanes. Punta Arenas.

MARTINIC, M. 2005. De la Trapananda al Áysen. Pehuén Editores, Santiago.

MARTINIC, M., A. PRIETO y P. CÁRDENAS. 1995. Hallazgo del asentamiento del Jefe Aónikenk Mulato en el valle del 
Zurdo. Una prueba de la sedentarización en el período histórico final. Anales del Instituto de la Patagonia Serie Ciencias Sociales 23:87-94.

MARTINIC, M. y A. PRIETO. 1986. Dinamarquero, encrucijada de rutas indígenas. Anales del Instituto de la Patagonia, Serie Ciencias Sociales 16:53-83.

MARTINIC, M. y A. PRIETO. 1988. Artesanía aónikenk sobre metal a la luz de hallazgos arqueológicos. Anales del Instituto de la Patagonia, Serie Ciencias Sociales 18:99-105.

MENA, F. 1998. Middle to late Holocene adaptations in Patagonia. Patagonia. Natural History, Prehistory and Ethnography at the uttermost end of the earth, editado por C. Mc Ewan, L. Borrero y A. Prieto, pp. 46-59. (eds.). London British Museum, London.

MENA, F. 2000. Un panorama de la prehistoria de Aisén Oriental; estado de conocimiento a fines de siglo. Serie Antropología 2:21-41.

MENA, F. y D. JACKSON. 1991. Tecnología y subsistencia en el Alero Entrada Baker, Región de Aisén, Chile. Anales del Instituto de la Patagonia 20:169-203.

MENA, F. y V. LUCERO. 2004. En torno a las últimas poblaciones indígenas de la cordillera centro-patagónica: estudio comparado de tres valles en Aisén oriental (Chile). En: Contra viento y marea. Arqueología de Patagonia. Actas de las V Jornadas de Arqueología de la Patagonia, editado por M. Civalero, P. Fernández y G. Guráieb, pp. 643-657. Instituto Nacional de Antropología y Pensamiento Latinoamericano y Sociedad Argentina de Antropología, Buenos Aires.

MÉNDEZ, C. y H. VELÁSQUEZ. 2005. Tecnología y subsistencia en Alero Entrada Baker: una revisión a la luz de nuevos antecedentes. Actas del XVI Congreso Nacional de Arqueología Chilena, pp. 593-602. Tomé, 2003.

MÉNDEZ, C., O. REYES y H. VELÁSQUEZ. 2007. Tecnología lítica en el alto río Cisnes (estepa extra andina de la XI Región de Aisén): primeros resultados. Boletín de la Sociedad Chilena de Arqueología 39 en prensa.

MOLINARI, R. y S. ESPINOSA. 1999. Brilla tú, diamante "loco"... En: Soplando en el viento... Actas de las Terceras Jornadas de Arqueología de la Patagonia, pp. 189-198. Universidad Nacional del Comahue e Instituto Nacional de Antropología y Pensamiento Latinoamericano, Neuquén y Buenos Aires.

ORQUERA, L. 1987. Advances in the archaeology of the Pampa and Patagonia. Journal of World Prehistory 1(4):333-413.
PALERMO, M. 1986. Reflexiones sobre el llamado "complejo ecuestre" en la Argentina RUNA XVI:157-178.

PALERMO, M. 1988. La innovación agropecuaria entre los indígenas pampeanos- patagónicos. Génesis y procesos. Anuario del IEHS III:43-90.

POLITIS, G., G. MARTÍNEZ y M. BONOMO. 2001. Alfarería temprana en sitios de cazadores-recolectores de la Región Pampeana (Argentina). Latin American Antiquity 12(2):167-181.

POLITIS, G. y P. MADRID. 2001. Arqueología pampeana: estado actual y perspectivas. En: Historia Argentina Prehispánica, editado por E. Berberián y A. Nielsen, pp. 737-814. Brujas, Córdoba.

RATTO, N. 1994. Funcionalidad v/s adscripción cultural: cabezales líticos de la margen norte del Estrecho de Magallanes. En: Arqueología de Cazadores-Recolectores. Límites, casos y aperturas, editado por J. Lanata y L. Borrero. Arqueología Contemporánea 5:105-120.

REYES, O., C. MÉNDEZ, H. VELÁSQUEZ y V. TREJO. 2006. Distribuciones espaciales y contextos arqueológicos de cazadores recolectores esteparios en el alto río Cisnes (XI Región de Aisén). Magallania 34(2):75-90.

ROJAS, J. 1996. Los niños cristaleros: trabajo infantil de la industria, Chile, 1880-1950. Centro de Investigaciones Diego Barros Arana, DIBAM, Santiago.

ROJAS, G. y J. ROJAS. 2000. Informe Preliminar sobre materiales de vidrio recolectados en Veta María, El Abra. Proyecto Arqueológico El Abra, Sector La Veta María, MAA Consultores, Santiago.

SCHIFFER, M. 1996. Formation processes of the archaeological record. University of Utah Press, Salt Lake City.

SENATORE, X. 1996. Tecnología cerámica en el área de Piedra del Águila, provincias de Río Negro y Neuquén. Prehistoria 2:127-145.

SILVEIRA, M. 1996. Alero Los Cipreses (Provincia del Neuquén, República Argentina). Arqueología, sólo Patagonia, Segundas Jornadas de Arqueología de la Patagonia, editada J. Gómez, pp. 107-118. Centro Nacional Patagónico, Puerto Madryn.

VELÁSQUEZ, H., F. MENA, V. TREJO, y O. REYES. 2005. Un Panorama Histórico y Arqueológico en la cordillera Aisenina. Transición Siglo XIX-XX. Werken 7:5-20.

VELÁSQUEZ, H., C. MÉNDEZ, O. REYES y V. TREJO. 2007. Analogía etnográfica y arqueología de tolderías en Patagonia central: El caso de Appeleg-1 (CIS 009), alto río Cisnes, XI Región de Aisén. Presentado al XVI Congreso Nacional de Arqueología, Valdivia. 2006. 